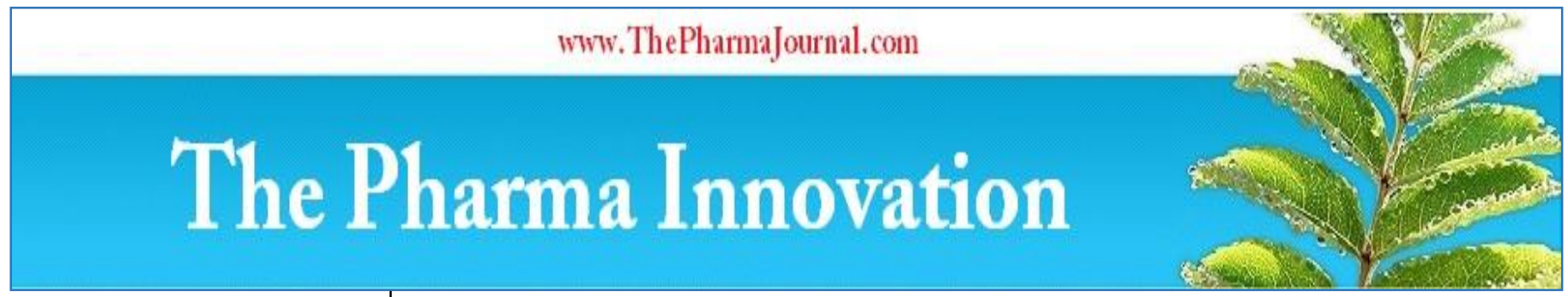

ISSN (E): 2277 - 7695

ISSN (P): 2349-8242

NAAS Rating: 5.03

TPI 2020; 9(9): 467-472

(C) 2020 TPI

www.thepharmajournal.com

Received: 07-07-2020

Accepted: 09-08-2020

\section{Dipanwita Das}

Department of Pathology, Orissa University of Agriculture and

Technology Bhubaneswar, Odisha, India

\section{Amrut Kumar Roul}

PG Department of Poultry

Science, Orissa University of

Agriculture and Technology

Bhubaneswar, Odisha, India

\section{Soubhagya Muduli}

PG Department of Poultry

Science, Orissa University of

Agriculture and Technology

Bhubaneswar, Odisha, India

Sudhanya Nath

Department of Animal

Nutrition, West Bengal

University of Animal and

Fishery Sciences Kolkata, West

Bengal, India

\section{Girija Prasanna Sabat}

Department of Livestock

Production and Management, Orissa University of Agriculture and Technology Bhubaneswar, Odisha, India

\section{Corresponding Author:} Dipanwita Das

Department of Pathology, Orissa

University of Agriculture and

Technology Bhubaneswar,

Odisha, India

\section{Immunomodulation in poultry}

\section{Dipanwita Das, Amrut Kumar Roul, Soubhagya Muduli, Sudhanya Nath and Girija Prasanna Sabat}

DOI: https://doi.org/10.22271/tpi.2020.v9.i9g.5167

\begin{abstract}
Immunity plays a key role in disease susceptibility. Immunomodulators are agents which specifically modulate immune system regulating immunity and disease resistance. In poultry it is specifically important as the growth, disease resistance, FCR, body weight gain production output mainly depends on the health and immunity of the chickens. Different types of immunomodulators are prebiotics, probiotics, vitamins, adjuvants, polysaccharides, herbs etc. Immunology has great potentials in prevention and treatment of various range of disorders for instance the various inflammatory diseases of skin, respiratory tract, gut, central organs and joints. The preliminary objective of immunomodulation is to improve host resistance to external and internal attacks by the microbes or other infectious agents. Immunomodulators can substitute many agents like antibiotics, antimicrobials etc. for the improvement of the immune system. They also enhance the qualities of feed and immune molecules enhancing all possibilities to fight diseases and maintain health homeostasis. Since, they are not popular and not included in the poultry feed on routine basis, more efforts should be made to popularize it.
\end{abstract}

Keywords: Immunomodulators, immunity, chicken, phytochemicals, probiotics, prebiotics

\section{Introduction}

Poultry sector of India ranks $1^{\text {st }}$ in the world. It has been a major source of income for rural farmers along with people making it a business. A major part of India's GDP is contributed by poultry industry. Around $70-80 \%$ of Indian people consume chicken, hence the health and growth of poultry (chicken) plays a major role in health of humans.

Immunomodulators are agents which modulate and regulate immune system affecting the immunity of the body. They are either natural or man induced. Immunity plays a crucial role in poultry farming. The growth, disease resistance, FCR, body weight gain etc everything depends directly and indirectly on the immunity of the bird. It also hence affects the profit of the farmer and the health of the consumer.

Immunomodulators modify and optimumly control immune responses of the body, modifying disease resistance, vaccine and antibiotic efficacy, improving digestive tract microflora. Nowadays, microbes are increasing resistance against antibiotics, hence immunomodulation can be a virtuous alternative prophylactic and therapy. Different types of immunomodulators are prebiotics, probiotics, vitamins, adjuvants, polysaccharides, herbs etc.

Immunomodulators have upper hand against antimicrobials. They act on the immune system and its molecules and not o the microbes directly, along with broad spectrum ability. Immunomodulators can come closer to the idea of one health one medicine concept. (Dhama et al., 2013) [7]

\section{Immunity system of chicken}

Immunology has great potentials in prevention and treatment of various range of disorders for instance the various inflammatory diseases of skin, respiratory tract, gut, central organs and joints. Moreover, infectious diseases are being primarily considered as immunological disorders. Neoplastic diseases along with numerous autoimmune diseases causes immunosuppression. (Patil et al., 2012)

Immunomodulators are either natural or synthetic substances which regulate and normalize the immune system. (Patil et al., 2012) Immunomodulators moderate immune response to help restore immunity towards treatment of diseases. (John Hadden, Immunomodulators) Hence, immunomodulators are also called as biological response modifiers (BRMs). 
immunomodulators rectify instable immune system. (Patil et al. 2012, John Hadden, Immunomodulators) The include microbial products, drugs, and proteins derived from the immune system (cytokine and antibodies) are currently used agents. (John Hadden, Immunomodulators). The cytokines (lymphokines and interleukins) are produced commonly by recombinant genetic methods in diverse vectors while the antibodies are produced by monoclonal antibody-producing cell cultures. most of these have anticancer activity directly. (John Hadden, Immunomodulators)

\section{Objectives of Immunomodulation}

The priliminary objective of immunomodulation is to improve host resistance to external as well as internal attacks by the microbes or other infectious agents. The basic objectives of immunomodulation in domestic animals include (Kuldeep Dhama et al. 2015) ${ }^{[31]}$ :

- To trigger powerful and sustained immune response against disease causing microorganism

- To improve maturation of specific and non specific immunity throughout neonatal period besides in susceptible young animals.

- To augment local protective immune reactions at susceptible sites or gastro intestinal tract.

- To overcome the immunosuppressive effects of stress and environmental pollution

- To enhance duration and level of immune response following vaccination

- To maintain immune surveillance

\section{Types of Immunomodulators}

Treatment immunomodulators are more potent and possibly cause aggrevated side effects than Natural immunomodulators. synthetic immunomodulator suppositories, for example azathioprine, methotrexate, 6mercaptopurine, and mycophenolate mofetil, function by the immune system suppression. The benefits of immunomodulators branch from their proficiency to accelerate defense mechanisms of natural and adaptive types, for instance cytokines, which empowers the body to assist itself.

Generally, two types of immunomodulators exist immunostimulants and immunosuppressants.

Immunosuppressants subdue the immune system hence used for pathological immune response control in autoimmune disease, etc. Immunostimulants are the mediators which are help enhance the body's resistance towards infections. Immunostimulants improve the basal levels of the immune response, and in individuals with impairment of immune response as an immunotherapeutic agent. immunodeficiency state, cancer, autoimmune disease, and viral infection can successfully be treated with immunostimulant drugs. (U.S Patil et al. 2012, John Hadden, Immunomodulators)

\subsection{Probiotics and Prebiotics}

Some species of non-pathogenic intestinal microbiota also interlink with the epithelium and immune system, modulating tissue physiology and ability to respond to infection. (John Hadden, Immunomodulators) Probiotics and prebiotics modifies the intestinal microbiota and immune system to lower pathogens colonization in definite conditions. Growth promoting antibiotics, stress status and environmental conditions influence effectiveness of prebiotics and probiotics. (John Hadden, Immunomodulators) These are spectacular alternatives for antibiotics used as growth promoters. (John Hadden, Immunomodulators)

\subsection{Phytochemicals}

Improving animal health and the GIT tract health are priorities in animal production other than reproduction. These are potential feed additives possessing multiple functions, including anti-inflammatory, antifungal, antiviral, and antioxidative properties. On the molecular level, it has impact of inflammatory gene expression on underlying mechanisms, similar to the effects of environmental stimuli along with phytochemicals in initiating nuclear factor kappa B and mitogen activated protein kinases signaling pathways improving health conditions. The active constituents in phytogenic, Phytochemicals, divided into two groups terpenes and terpenoids, or aromatic and aliphatic compounds, on the different synthetic pathways in plants: Phytochemicals can be used as alternative feed additives. Addition of phytochemicals had tremendous effects on the pancreatic and intestinal enzyme secretion in broilers and improved dry matter along with crude protein digestibility. immune response improved besides GIT protective capacity against exogenous stressors, when phytogenic was introduced in diet. (John Hadden, Immunomodulators) Their major components regulates $\mathrm{NF} \kappa \mathrm{B}$ (nuclear factor enhancing kappa light chains of activated B cells) and MAPKs (mitogen-activated protein kinase) signaling pathways in rodents (John Hadden, Immunomodulators) Therefore, phytochemicals regulate the immune response and moderate the expression of inflammatory mediators in chickens (John Hadden, Immunomodulators). The stress response was alleviated as the $\mathrm{NF \kappa B}$ and MAPKs signaling pathways were suppressed, also the expression of anti-inflammatory cytokines increased due to this, the blood levels of nonspecific immune cells like heterophils (neutrophils) reduced, and lymphocyte and antibody production increased to defend against invading pathogens. The lesser energy spent by chickens on the nonspecific immunity, the more energy is available for production and growth. Phytochemicals modulate NFkB and/or MAPKs signaling pathways in chickens, but detailed mechanisms, expression of upstream molecules involving signalling of NFאB and MAPKs and the relationship between antioxidant and antiinflammation, are still unknown.

\subsection{Turmeric Rhizome Powder (TRP)}

TRP in the diet significantly increased blood IgA, IgG, and IgM levels, and decreased the ratio of monocytes in 42dayold broilers given SRBCs (sheep red blood cells) (Emadi M, et al. 2007) ${ }^{[29]}$. $0.2 \%$ TRP supplementation increased the total secondary antibody titer against SRBCs in broilers and decreased the heterophils and lymphocytes ratio $(\mathrm{H} / \mathrm{L})$ under heat stress treatment (Akhavan-Salamat H2016) ${ }^{[27]}$. The H/L ratio denotes stress level Treatment of $0.33 \%, 0.66 \%$, and $1.0 \%$ TRP reduced abdominal fat content and serum triglyceride concentration. (Nouzarian R2011) ${ }^{[28]}$ hence, dietary curcumin regulates immune responses in poultry and it has the potential to enhance growth performance.

\subsection{Cinnamon}

Dietary supplementation of cinnamon extracted oil or leaves has shown to decrease coliform bacteria in the jejunum and large intestine and improve growth performance in broilers. inclusion of $0.4 \%$ and $0.8 \%$ cinnamon in broiler diets improved the FCR and (C. M. Huang, 2018) [26] the 
proportion of lymphocytes in the blood and increased the concentration of hemoglobin; Cinnamon essential oil has antioxidant properties (Case and his colleagues 1995; lee and his colleagues 2001; Yu and his colleagues 2002; Lee and his colleagues 2007).

The response of cinnamon powder or oil on humor and innate immunity responses is significant. Phagocytosis and lysosomal activity, host resistances mortality (Balekar et al. 2014) suggested that PP-CZ (polyphenol cinnamon) treatments was effective immunostimulant in immune system in dos dependent manner. Peripheral PMN was increased by PP-CZ treatment along with increase in the number of resident peritoneal macrophages in mice, $\mathrm{pp}-\mathrm{CZ}$ stimulates non-specific immunity as phagocytosis activity in mice improved by increasing the number of macrophages and phagocytic activity in mice on sub-acute treatment. A dosedepedent trend for increased numbers of peritoneal macrophages was observed and increased survival rate in mice. There are specialized phagocytic cells that cells attack foreign subsatnces, infectious microbes through destruction and ingestion [Ovchinnikov DA., 2008] [9]. [Balekar et al. Journal of Applied Pharmaceutical Science 4(07); 2014:114122] demonstrated sub-acute treatment of PP-CZ showed increased peritoneal macrophage and increased PMN observed in their study the potential PP-CZ in stimulating adaptive immunity against infectious pathogens. [A. Sivagurunathan et al. 2014] [3] demonstrated significant increase in TLC can be considered as indicator for improving general resistance, increase in neutrophil, non-specific immune respond and increase in lymphocytes counts in cinnamon along with specific immune response. Cinnamon verum essential oil showed stimulator effect on macrophages, phagocytosis and killing of invading microorganisms by macrophage constitute the body's primary line of defense against infection Van furt R (1982) (Tamam S. M et al. 2017).

\subsection{Thyme}

Thyme is the name for Thymus; thymol, carvacrol, pcymene and $\gamma$ terpinene are the major active components in the essential oil. Supplementation is in the form of essential oil or leaf powder. this essential oil improved cutaneous basophil hypersensitivity to phytohaemagglutinin P (PHAP) along with reducing the $\mathrm{H} / \mathrm{L}$ ratio. It produced more balanced intestinal microbiology, indicated by higher Lactobacillus and Bifidobacterium counts and lower Escherichia coli (E. coli) levels in the ileum. A reduced $\mathrm{H} / \mathrm{L}$ ratio shows enhanced immunoregulatory capacity of the bird. Intestinal microbiota plays vital role in the immune system of chickens. Thyme improved body weight gain and FCR in broilers. Hence, thyme is a potent feed additive in poultry production.

\subsection{Essential oils and Carvacrol}

Essential oils (EOs) are vital aromatic components, which are used as natural substitutes for antibiotic growth promoters (AGPs) in poultry feed. These have antimicrobial, antiviral, antifungal, antiparasitic properties. Other benefits are, it acts as appetite stimulant, improves enzyme secretion related to food digestion, and immune response activation.

Carvacrol, or cymophenol is monoterpoid phenol having characteristic pungent, (16)

Carvacrol is found in the essential oil of Origanum vulgare (oregano), oil of thyme, oil of pepperwort, and wild bergamot. (Vladić, J. 2016) ${ }^{[24]}$. EOs are comprised of mainly two classes of compounds- terpenes and phenylpropenes.
The antimicrobial properties of the various chemical compounds of EOs has a cumulative effect on various targets in different cell parts (Burt, 2004) ${ }^{[19]}$. The effectiveness depends on $\mathrm{pH}$, concentration, chemical structure, or the individual bioactive compound, along with the affected microorganisms types and population. The antimicrobial mechanisms comprise of different activities, like membrane disruption by phenolics and terpenoids, metal chelation by flavonoids and phenols, and effect on genetic material by coumarin and alkaloids due to inhibition of microorganisms growth (Cowan, 1999) ${ }^{[20] .}$

EOs are more effective against Gram-positive in comparison to Gram-negative pathogens (Burt, 2004) ${ }^{[19]}$ as the outer membrane through its lipopolysaccha-ride structures limits the intrusion of hydrophobic compounds (Vaara, 1992) ${ }^{[21] .}$ Many EOs increase beneficial microbe growth and control pathogenic bacteria population in poultry (Wenk, 2000) ${ }^{[11]}$. Cerisuelo et al. (2014) ${ }^{[12]}$ showed a clear effectiveness of low doses of EOs and sodium butyrate in Salmonella control in broilers.

EOs enhance the growth of beneficial microbes and limit the number of pathogenic bacteria in poultry (Wenk, 2000) ${ }^{[11]}$. Cerisuelo et al. (2014) ${ }^{[12]}$ showed clear effectiveness of low doses of EOs and sodium butyrate in Salmonella control in broilers. Thymol and Carvacrol, are the main ingredients in oregano oil, possessing anticoccidial actions against mixed Eimeria spp. and E.tenella infection (Oviedo-Rondón, 2003) (Giannenas et al., 2003) ${ }^{[4]}$. several in vitro and in vivo studies suggested that phenols can be used against E. tenella as ocysticides (Williams, 1997) ${ }^{[23]}$.

Several EOs are antiparasitic in function as well. EOs and seeds of onion (Allium cepa), garlic (Allium sativum), and mint (Mentha spp.) are effective against gastrointestinal parasitism. EO also suppress inflammatory prostaglandins metabolism. (Craig, 2001) ${ }^{[1]}$. plants with anti-inflammatory potentials are licorice, chamomile, marigold, and anise (Srinivasan, 2005 (Gopal Krishan and Asmita Narang, 2014) [10].

\subsection{Butyrate}

Butyrates stimulates digestive secretions (bile and mucus) while enhancing enzymatic activity (Platel, 2004; Manzanilla et al, 2004). In broilers, EO (essential oils) boost trypsin, amylase and jejunal chyme secretions (Jang et al., 2007) ${ }^{[15]}$, as well as reduces the pathogens adherence (for example, E. coli and $\mathrm{Cl}$. perfringens) to intestinal wall (Jamroz et al., 2006). (Gopal Krishan and Asmita Narang, 2014) ${ }^{[10]}$

\subsection{Nutritional Strategies}

Reduced feed intake is observed at high environmental temperature. It causes micronutrients deficiency like that of vitamins and minerals. Vitamin A, C and E in heat-stressed chicken has stress alleviation and immunomodulation role.

\subsubsection{Vitamin A}

Fat soluble vitamin A has significant role in immunity. it reduces heat stress When layer supplemented @ 8000 IU/Kg. diet, along with increased egg production. in broilers, when supplemented @15000 IU/Kg. diet, significant improved weight gain with, feed efficiency and carcass quality is reported. in combination with zinc it is more effective to minimize heat stress.

\subsubsection{Vitamin C}


Ascorbic acid is a water soluble vitamin and can be synthesized in poultry and not required as a supplement under normal condition. However, in heat stressed chicken, it is proven to be beneficial by reducing corticosterone production with simultaneous increase in insulin and thyroid levels. It also deprives protein derived glucose synthesis and increase fat degradation. In case of broiler breeders, it increases egg weight, fertility and hatchability. In heat stressed broilers, ascorbic acid requirement is more, as it also acts as antioxidant. It improves carcass yield and quality, feed efficiency, carcass protein content and reduces crude fat.

\subsubsection{Vitamin $\mathrm{E}$}

Vitamin $\mathrm{E}$ is a fat-soluble vitamin and its dietary intake reduces heat stress and improves egg production. It increases yolk and albumen contentin the egg. It increases plasma concentration of yolk lipids by production and release from liver. It protects the liver and body cells from oxidative injuries also. Vitamin E supplementation @ $250 \mathrm{mg} / \mathrm{Kg}$. diet is beneficial for egg production at high temperature. Vitamin E shall be supplemented before, during and after any stressful condition.

\subsubsection{Herbal plants and HSPs}

Herbal plants and derivatives like secondary metabolites include saponins, polysaccharides, glyacoproteins, and flavonoids etc are being used for therapeutic and immunomodulatory functions. Aloe vera, Panax ginseng, Agelicagiggas, Gingiber officinale, and Saussurealappacan are few other herbs. Many of these are used as the immunomodulators, tonics and adaptogens. These metabolites improve lymphocyte stimulation.

\subsubsection{Minerals and others}

Chromium, being essential trace mineral improves feed intake, feed efficiency, body weight and carcass characteristics when supplemented @ $120 \mathrm{ppb} / \mathrm{Kg}$ of diet. It substitutes for insulin levels and serum cortisol deprivation. Chromium reduces heat stress in birds. of chromium is in biological system one. Cr-Nano particles are comparatively effective than Cr-Macroparticles having greater absorption rate in the gut. Whereas Organic form is more active than inorganic form. it improves leucocytic activity and antibody response. Many feed additives like essential oil mixtures, polyherbal preparations, probiotics, betaine, acetic acid, aspirin, and prebiotics alleviates heat stress and potentiates chicken immunity and is very promising in enhancing heat tolerance of birds.

\section{Conclusion}

Immunomodulators can substitute many agents like antibiotics, antimicrobials etc. for the improvement of the immune system. As immune system is directly related to the disease resistance, gut health, FCR, etc., hence they must be used in combination and/or alone according to the needs. They also improve the qualities of feed and immune molecules enhancing all possibilities to fight diseases and maintain health homeostatsis. However, they are not popular and not included in the poultry feed on routine basis. Hence, more studies should be done and efforts should be made to popularise it.

\section{References}

1. Craig WJ. Herbal remedies that promote health and prevent disease. In: Watson RR (Ed.). Vegetables, fruits, and herbs in health promotion. CRC Press, Boca Raton, Florina, USA, 2001, 179-204.

2. Garcia Manzanilla E, Nofrarías M, Anguita M, Castillo M, Pérez J, Martín-Orúe S et al. Effects of butyrate, avilamycin, and a plant extract combination on the intestinal equilibrium of early-weaned pigs. Journal of animal science. 2006; 84:2743-51. 10.2527/jas.2005-509.

3. Sivagurunathan B. Xavier Innocent. Immunomodulatory. Effect of Dietary Cinnamon in Growth and Haematology of Tilapia Challenged with Pseudomonas Aeruginosa Int. J Pharm. Phytopharmacol. Res. 2014; 3(4):277-280.

4. Giannenas I, Florou-Paneri P, Papazahariadou M, Christaki E, Botsoglou NA, Spais AB. Effect of dietary supplementation with oregano essential oil on performance of broilers after experimental infection with Eimeria tenella, Archives of Animal Nutrition. 2003; 57(2):99-106,

DOI: $10.1080 / 0003942031000107299$

5. Oviedo E, Clemente-Hernandez S, Salvador F, Williams P, Losa R. Essential Oils on Mixed Coccidia Vaccination and Infection in Broilers. International Journal of Poultry Science. 2006; 5:10.3923/ijps.2006.723.730.

6. John W Hadden. Immunomodulators. Immunopharmacology Reviews 2,3-2

7. Dhama K, Saminathan M, Jacob SS, Singh M, Amarpal KK, Tiwari $\mathrm{R}$ et al. Effect of Immunomodulation and Immunomodulatory Agents on Health with some Bioactive Principles, Modes of Action and Potent Biomedical Applications. International Journal of Pharmacology. 2015; 11:253-290.

8. Abdel-Hameed, Mohamed, Helmy, Samah, Nakhriry, Marwa. The Anti-viral and Immunomodulatory Activity of Cinnamon zeylanicum Against "NDV" Newcastle Disease Virus in Chickens. International Journal of Sciences: Basic and Applied Research (IJSBAR). 2017; 32:251-262.

9. Ovchinnikov DA. Macrophages in the embryo and beyond: much more than just giant phagocy Genesis. 2008; 46:447-62

10. Krishan, Gopal, Narang, Asmita. Use of essential oils in poultry nutrition: A new approach. Journal of Advanced Veterinary and Animal Research, 2014, 1(1). 10.5455/javar.2014.a36.

11. Wenk C. Recent Advances in Animal Feed Additives such as Metabolic Modifiers, Antimicrobial Agents, Probiotics, Enzymes and Highly Available Minerals Review -Asian-Australas J Anim Sci. 2000; 13(1):86-95. DOI: https://doi.org/10.5713/ajas.2000.86

12. Cerisuelo C, Marín F, Sánchez-Vizcaíno EA, Gómez JM, de la Fuente R, Durán C, et al, The impact of a specific blend of essential oil components and sodium butyrate in feed on growth performance and Salmonella counts in experimentally challenged broilers, Poultry Science, 2014; 93(3):599-606, ISSN 0032-5791, https://doi.org/10.3382/ps.2013-03528.

13. Jamroz D, Wertelecki T, Houszka M, Kamel C. Influence of diet type on the inclusion of plant origin active substances on morphological and histochemical characteristics of the stomach and jejunum walls in chicken. Journal of Animal Physiology and Animal Nutrition, 2006; 90:255-268. doi:10.1111/j.14390396.2005.00603.x

14. Jamroz D, Wertelecki T, Houszka M, Kamel C. Influence 
of diet type on the inclusion of plant origin active substances on morphological and histochemical characteristics of the stomach and jejunum walls in chicken. J Anim. Physiol. Anim. Nutr. 2006; 90:255-268.

15. Jang I, Ko Y, Kang S, Lee C. Effect of a commercial essential oil on growth performance, digestive enzyme activity and intestinal microflora population in broiler chickens. Animal Feed Science and Technology - ANIM FEED SCI TECH. 2007; 134:304-315. 10.1016/j.anifeedsci.2006.06.009.

16. Hadden JW. Immunomodulators. In: Hadden J.W., Szentivanyi A. (eds) Immunopharmacology Reviews 1996, 2. Springer, Boston, MA https://doi.org/10.1007/978-1-4613-0349-7_1

17. Parveen P, Usha P, Sowjanya Ch, Sravika SVRL, Bhavana Ragala, Gayatri Ramya M. An Overview of Immunology - Systemic Review. American Journal of Phytomedicine and Clinical Therapeutics. 2013. ISSN 2321 - 2748. AJPCT[1][8][2013]628-644

18. Patterson JA, Burkholder KM. Application of Prebiotics and Probiotics in Poultry Production. 2003 Poultry Science. 2003; 82:627-631

19. Burt S. Essential oils: their antibacterial properties and potential applications in foods - a review. International journal of food microbiology. 2004; 94:223-253.

20. Cowan MM. Plant products as antimicrobial agents. Clinical Microbiology Review. 1999; 12:564-582.

21. Vaara M. Agents that increase the permeability of the outer membrane. Microbiology Review, 1992; 56:395411.

22. Oviedo-Rondón EO, Hume ME, Hernández C, ClementeHernández S. Intestinal microbial ecology of broilers vaccinated and challenged with mixed Eimeria species, and supplemented with essential oil blends. Poultry Science, 2006; 85:854-860.

23. Williams RB. Laboratory tests of phenolic disinfectants as oocysticides against the chicken coccidium Eimeria tenella. Veterinary Record. 1997; 141:447-448.

24. Patterson JA, Burkholder KM. Application of prebiotics and probiotics in poultry production. Poult Sci. 2003; 82(4):627-631. doi:10.1093/ps/82.4.627.

25. Vladić J, Zeković Z, Jokić S, Svilović S, Kovačević S, Vidović S. "Winter savory: Supercritical carbon dioxide extraction and mathematical modeling of extraction process". The Journal of Supercritical Fluids. 2016; 117:89-97. doi:10.1016/j.supflu.2016.05.027

26. Ultee A, Slump RA, Steging G, Smid EJ. "Antimicrobial activity of carvacrol toward Bacillus cereus on rice". Journal of Food Protection. 2000; 63(5):620624. doi:10.4315/0362-028x-63.5.620. PMID 10826719.

27. Huang $\mathrm{C}$, Lee $\mathrm{T}$. Immunomodulatory effects of phytogenics in chickens and pigs - A review AsianAustralas J Anim Sci 2018; 31(5):617-627. DOI: https://doi.org/10.5713/ajas.17.0657

28. Akhavan-Salamat $H$, Ghasemi HA. Alleviation of chronic heat stress in broilers by dietary supplementation of betaine and turmeric rhizome powder: dynamics of performance, leukocyte profile, humoral immunity, and antioxidant status. Trop Anim Health Prod. 2016; 48:1818

29. Nouzarian R, Tabeidian SA, Toghyani M, Ghalamkari G, Toghyani M. Effect of turmeric powder on performance, carcass traits, humoral immune responses, and serum metabolites in broiler chickens. J Anim Feed Sci. 2011;
20:389-400.

30. Emadi M, Kermanshahi H. Effect of turmeric rhizome powder on immunity responses of broiler chickens. J Anim Vet Adv. 2007; 6:833-6

31. Immunomodulation in poultry under stress conditions http://www.pashudhanpraharee.com/immunomodulationin-poultry-under-stress-conditions/

32. Kuldeep Dhama, Mani Saminathan, Siju Susan Jacob, Mithilesh Singh, Karthik K, Amarpal, et al. Effect of Immunomodulation and Immunomodulatory Agents on Health with some Bioactive Principles, Modes of Action and Potent Biomedical Applications. International Journal of Pharmacology, 2015; 11:253-290.

33. Keestra AM, de Zoete MR, Bouwman LI, Vaezirad MM, Van Putten JPM. Unique features of chicken toll-like receptors. Dev. Comp. Immunol. 2013; 41:316-323. https://doi.org/10.1016/j.dci.2013.04.009

34. Ganz T. Defensins: antimicrobial peptides of innate immunity. Nat. Rev. Immunol. 2003; 3:710-720. https://doi.org/10.1038/nri1180

35. Derache C, Labas V, Aucagne V, Meudal H, Landon C, Delmas AF et al. Primary structure and antibacterial activity of chicken bone marrow-derived $\beta$-defensins. Antimicrob. Agents Chemother. 2009; 53:4647-4655. https://doi.org/10.1128/AAC.00301-09

36. Miller MM, Taylor RL. Brief review of the chicken major histocompatibility complex: the genes, their distribution on chromosome 16, and their contributions to disease resistance. Poult. Sci. 2016; 95:375-392. https://doi.org/10.3382/ps/pev379

37. Kaiser P, Poh TY, Rothwell L, Avery S, Balu S, Pathania US, Hughes $\mathrm{S}$ et al. A genomic analysis of chicken cytokines and chemokines. J. Interferon. Cytokine Res. 2005; 25:467-484. https://doi.org/10.1089/jir.2005.25.467

38. Warr GW, Magor KE, Higgins DA. IgY: clues to the origins of modern antibodies. Immunol. Today 1995; 16:392-398.

https://doi.org/10.1016/0167-5699(95) 80008-5.

39. Kogut MH, Rothwell L, Kaiser P. IFN- $\gamma$ Priming of Chicken Heterophils Upregulates the Expression of Proinflammatory and Th1 Cytokine mRNA Following Receptor-Mediated Phagocytosis ofSalmonella enterica serovar enteritidis. J. Interferon. Cytokine Res. 2005; 25:73-81. https://doi.org/10.1089/jir.2005.25.73

40. Cooper M, Peterson R, Good R. Delineation of the Thymic and Bursal Lymphoid Systems in the Chicken. Nature 1965; 205:143-146.

41. Lillehoj HS, Trout JM. Avian gut-associated lymphoid tissues and intestinal immune responses to Eimeria parasites. Clin. Microbiol. Rev. 1996; 9:349-360

42. Guglani L, Khader SA. Th17 cytokines in mucosal immunity and inflammation. Curr. Opin. HIV AIDS 2010; 5:120-127.

https://doi.org/10.1097/COH. 0b013e328335c2f6

43. Min W, Kim WH, Lillehoj EP, Lillehoj HS. Recent progress in host immunity to avian coccidiosis: IL-17 family cytokines as sentinels of the intestinal mucosa. Dev. Comp. Immunol. 2013; 41:418-428. https://doi.org/10.1016/j.dci.2013.04.003.

44. Kim WH, Jeong J, Park AR, Yim D, Kim YH, Kim KD et al. Chicken IL-17F: identification and comparative expression analysis in Eimeria-infected chickens. Dev. Comp. Immunol. 2012; 38:401-409. 
https://doi.org/10.1016/j.dci.2012.08.002.

45. Kim WH, Jeong J, Park AR, Yim D, Kim S, Chang HH, et al. Downregulation of chicken interleukin-17 receptor A during Eimeria infection. Infect. Immun. 2014; 82:3845-3854. https://doi.org/10.1128/IAI.02141-14.

46. Williams RB. Laboratory tests of phenolic disinfectants against the chicken coccidium Eimeria tenella. Veterinary Record. 1997b; 141:447-448. 